Results: 15 patients were included: 11 women and 4 men, with a mean age of 42.2 years $(S D+/-16)$

The genetic study was performed in 12 patients: $75 \%$ were positive for HLA DQ2 and $25 \%$ for HLA DQ8.

The determination of antibodies was positive in 12 patients (73\%) and negative in $3(27 \%)$. These 3 were positive for the genetic study (2 HLA DQ2 and 1 HLA DQ8).

The study of BD showed: type I in 4 patients; Type IIla in 2 patients; Type IIIb in 6 patients; Type IIIC in 3 patients.

BMD showed normal values in $33 \%$, osteopenia in $47 \%$ and osteoporosis (OP) in $20 \%$ of patients. Thus, $67 \%$ had an alteration in BMD at the time of diagnosis.

$100 \%$ had a Vit D deficit, with a mean value of $14.3 \mathrm{ng} / \mathrm{mL}$ (range $4.15-26.8$ ). $75 \%$ of them had values below $20 \mathrm{ng} / \mathrm{mL}(40 \%$ less than $10 \mathrm{ng} / \mathrm{mL})$.

$40 \%$ had secondary hyperparathyroidism (SHP), $83 \%$ of which had an alteration in BMD (67\% osteopenia and $16 \%$ OP). The BD of the patients with SHP and alteration of the BMD showed a histological pattern type Marsh III.

No patient presented an alteration in the values of calcemia, phosphataemia or calciuria.

The relationship between the degree of alteration in BMD and the Marsh classification (table) was studied.

$72 \%$ of patients with a Marsh III had an alteration in BMD (3 OP and 5 osteopenia vs 3 normal).

\begin{tabular}{lccccc}
\hline DMO & Marsh I & Marsh Illa & Marsh IIIB & Marsh IIlc & Total \\
\hline Osteoporosi & 0 & 0 & 2 & 1 & 3 \\
Osteopenia & 2 & 2 & 1 & 2 & 7 \\
Normal & 2 & 0 & 3 & 0 & 5 \\
Total & 4 & 2 & 6 & 3 & 15 \\
\hline
\end{tabular}

Conclusions: Most patients with celiac disease have an alteration in BMD. In our study, all patients with celiac disease had a Vit D deficiency with no alteration of calcemia or calciuria.

Only in patients with SHP, there is a correlation between alterations in BMD and a higher degree of Marsh.

Most patients with a histological Marsh III have abnormalities in BMD.

Disclosure of Interest: None declared

DOI: 10.1136/annrheumdis-2017-eular.3114

\section{AB0844 FALLS: RISK AND PREVENTION IN PRIVATE RHEUMATOLOGY PRACTICE}

L. Poulain ${ }^{1}$, V. Strotz ${ }^{2}$, X. Grapton ${ }^{3}$, P. Lemesle ${ }^{4}$ on behalf of Groupe CREER. ${ }^{1}$ Private Rheumatology Practice, la Garenne-Colombes; ${ }^{2}$ Private Rheumatology Practice, Antony; ${ }^{3}$ Private Rheumatology Practice, Colombes; ${ }^{4}$ Private Rheumatology Practice, Bois-Colombes, France

Background: Menopaused and osteoporotic women have a higher risk of fractures when falling. Fall prevention is important when taking care of those women.

Objectives: After identifying fall risk factors, defining the \% of fractured fallers and the part of osteoporotic women in this population, we want to evaluate the relevance of balance tests and to favor prevention measures for those patients.

Methods: 110 patients, 60 years and older (including 24 controls), having falled within a year, have seen 28 private practice rheumatologists in the larger Paris area and were subject of our multicentre retrospective study.

Results: Fallers mean age was 75 years.

$37 \%$ of the fallers within a year fractured after falling. Among them, $95 \%$ were post-menopausal fractures versus $64 \%$ when including the control group.

Post-fall or post menopausal fracture sites are identical. In order: spine $26 \%$, wrist $18 \%$, ribs $6 \%$.

The circumstances of the falls are: 1) lack of attention 2) slipping 3) stumbling $61 \%$ of the fallers have at least one physical activity, among them $71 \%$ a weight-bearing physical activity. The most common physical activity was walking (46\%).

Among the fractured, $32 \%$ had physical activity, $42 \%$ hadn't.

$72 \%$ of the fallers had at least one fall risk factor: $94 \%$ had intrinsic risk factors, $28 \%$ extrinsic, and an average of 2 risk factors within the older than 80 years.

Intrinsic factors: 1) osteo-articular 2) eye-vision 3) postural 4) drugs, out of 13 items.

Extrinsic: 1) obstacles 2) footwear out of 7 items.

$45 \%$ of the fallers within a year had at least one osteoporosis risk factor: 1) low body mass index

2) cortico-steroids 3) early menopausis 4) smoking, out of 9 items.

$70 \%$ of our patients had vitamin D level $>30 \mathrm{ng} / \mathrm{l}$ (as recommanded)

$69 \%$ of the patients had recent bone density measures: bone density was lower only at femoral neck of fractured fallers versus non fractured fallers.

The unipodal balance test (positive when standing less than 5 seconds) was relevant in $42 \%$ of the patients with or without fractures. Get up and go test was not relevant in our study population.

$94 \%$ of the rheumatologists participating in the study recommend at least 2 fall prevention measures: 1 ) correct vitamin D levels 2 ) physical activity 3 ) reeducation 4) eye vision correction, out of 12 items.

Conclusions: A significant part of menopaused women with anteriority of falls and fractures will refracture after falling again. Beside treating osteoporosis, we should identify potential fallers by doing unipodal tests, consider and correct fall risk factors, encourage those patients to keep on physical activity and prescribe reeducation.

Disclosure of Interest: None declared

DOI: 10.1136/annrheumdis-2017-eular.1540

\section{AB0845 TECHNETIUM-99 CONJUGATED WITH METHYLENE DIPHOSPHONATE AMELIORATES GLUCOCORTICOID INDUCED OSTEOPOROSIS IN RATS}

L. Shi ${ }^{1}$, Y. Ning ${ }^{2}, \mathrm{~J} . \mathrm{Li}^{1}$, F. Hu${ }^{2}$, X. Zhang ${ }^{2} .{ }^{1}$ Peking University International Hospital; ${ }^{2}$ Peking University People's Hospital, Beijing, China

Background: ${ }^{99}$ Tc-MDP has been used in Chinese RA patients for over 15 years and many more studies have focused on this agent in RA. However, the previous studies limited in its effect on the effects of anti-inflammation. As a special form of bisphosphonate, the anti-osteoporotic effect of ${ }^{99} \mathrm{Tc}-\mathrm{MDP}$ is unclear.

Objectives: To systematically investigate the effect of technetium-99 conjugated with methylene diphosphonate ( $\left.{ }^{99} \mathrm{Tc}-\mathrm{MDP}\right)$, an anti-inflammatory drug effective in treating rheumatoid arthritis (RA), on cortical and cancellous bones in glucocorticoid-induced osteoporotic (GIO) rats, as well as comparing the effect of ${ }^{99}$ Tc-MDP with that of methylene diphosphonate (MDP).

Methods: Forty-eight Sprague-Dawley rats were randomly divided into six groups: blank, negative control, high dose, medium dose, low dose, and positive control groups. After dexamethasone was given to all groups except the blank group to induce osteoporosis, the rats in different groups were treated with saline, MDP, or different doses of

Results: Micro-CT analyses showed that: (1) ${ }^{99}$ Tc-MDP reversed glucocorticoid induced bone microarchitecture destruction by increasing vBMD, BV/TV, Tb.Th, Tb.N and decreasing BS/BV, Tb.Sp and TBPf; (2) effect of ${ }^{99}$ Tc-MDP increased as its dosage increased; and (3) ${ }^{99} \mathrm{Tc}-\mathrm{MDP}$ could improve cortical bone thickness while MDP failed to do so. Micro-CT spatial structure analysis and histology also yielded consistent results, indicating ${ }^{99} \mathrm{Tc}-\mathrm{MDP}$ increased trabecular number and connectivity morphologically. Finally, biomechanics revealed ${ }^{99} \mathrm{Tc}-\mathrm{MDP}$ can enhance the extrinsic stiffness of bone by changing bone geometry.

Conclusions: ${ }^{99} \mathrm{Tc}-\mathrm{MDP}$ has anti-osteoporotic effect by improving both cancellous and cortical bone, as well as increasing extrinsic bone stiffness. The dual effect of ${ }^{99} \mathrm{Tc}-\mathrm{MDP}$, anti-inflammatory and anti-osteoporotic, may suggest a potential clinical application for patients with both GIO and RA.

References:

[1] Hoes JN, Jacobs JW, Buttgereit F, et al. Current view of glucocorticoid cotherapy with DMARDs in rheumatoid arthritis. Nature reviews Rheumatology 2010:6:693-702.

[2] Bijlsma JW. Disease control with glucocorticoid therapy in rheumatoid arthritis. Rheumatology 2012;51 Suppl 4:iv9-13.

Acknowledgements: We thank Mr. Kai Gao for providing the rats and Dr. Min Feng for assistance in histological processing of the rats. This work was supported by grants from the Natural Science Foundation of China (81501396 to Dr. Lianjie Shi, and 81302554 to Dr. Fanlei Hu).

Disclosure of Interest: None declared

DOI: 10.1136/annrheumdis-2017-eular.6240

\section{AB0846 OSTEOPOROSIS AND ATHEROSCLEROSIS - ALTERNATIVE OR COEXISTENCE}

\section{Lagvilava. Rheumatology, Clinic "Consilium Medulla", Tiblisi, Georgia}

Background: The majority of scientific research data promote the idea that $\mathrm{OP}$ and Atherosclerosis are inter-connected via OPG system. Scientists have speculation that OPG is the molecular bond between artery hardening and bone resorbtion. Thus the mechanism explained above makes obvious the co-existence of two: Artery hardening and Osteoporosis.

Objectives: There are number of ongoing Clinical trials on Denosumab therapy in patients with diagnosis of Osteoporosis and Atherosclerosis. The increase of Bone mineral density is associated with less intensive hardening of arteries. This fact inspired us to study bone mass in patients with cardiovascular events and atherosclerosis.

Methods: 1675 men, age range $38-78$ years, mean age $59 \pm 4$.3 Disease duration $6,4 \pm 1,75$ with the diagnosis of Atherosclerosis (revealed on coronarography, assessed lipid profile).At the moment of research Stabile Angina Pectoris (Stable Angina) found in 20\% (335); unstable angina non S-T elevation in $48 \%$ (804); Myocardial Infarction 32\% (536) Bone Mass was assessed by DXA Absorbtiometry technique (Hologic 1000) using T and Z Scores (WHO 1994). As a control group 680 healthy Georgian men 40-70 age range were assessed.

Results: Men having atherosclerosis are rather predisposed to Osteoporosis than health individuals. In Atherosclerosis subgroup normal bone mass was measured in 23\% (385.25 patients) of patients; Osteopenia was diagnosed in 19\% (315.25 patients), Osteoporosis was detected in $58 \%$ (971.5\%) according to T-Score SD BMD records are shown in table 1.

Conclusions: 1 .According to our research more than $50 \%$ of men with verified Atherosclerosis are diagnosed to have Osteoporosis 2.The lowest BMD values were observed in Lumbar Spine L1-L4, indicating that trabecular bone is more 